\title{
Molecular characterization and pathogenicity of Streptococcus agalactiae serotypes Ia ST7 and III ST283 isolated from cultured red hybrid tilapia in Malaysia
}

\begin{abstract}
Streptococcus agalactiae is one of the most important pathogens infecting tilapia worldwide. In this study, we determined the serotype, sequence type (ST), virulence gene profile and pathogenicity of S. agalactiae isolated from cultured fish. A collection of $256 \mathrm{~S}$. agalactiae that were previously isolated from outbreaks of streptococcosis in red hybrid tilapia in Peninsular Malaysia were studied. The genotype and ST of the isolates were determined by molecular serotyping of the capsular polysaccharide (cps) gene clusters, and multi-locus sequence typing (MLST). The virulence gene profiles were constructed using m-PCR for 14 standard virulence genes with published primers. Two serotypes, with different associated STs, were identified in this study: serotypes Ia ST7 and III ST283. Serotype Ia ST7 lacks lmb, scpB, pavA, fbsB, cyl, bca, cspA and bac genes, which were present in serotype III ST283. The LD50-240h of S. agalactiae in red hybrid tilapia for serotypes Ia ST7 and III ST283 were $8.7 \times 103 \mathrm{CFU} / \mathrm{mL}$ and $6.3 \times 103 \mathrm{CFU} / \mathrm{mL}$, respectively. The histopathological findings were consistent with meningitis, necrosis of hepatocytes, coagulative tubular necrosis and hypocellular of the spleen. Histopathological scores revealed significant differences $(p<0.05)$ between the two serotypes in all lesions in the brain, liver (except hepato-necrosis) and spleen, but not the kidney. This study revealed that $\mathrm{S}$. agalactiae serotypes Ia ST7 and III ST283 currently exist in cultured tilapia in Malaysia. This molecular epidemiology study could be useful for the future development of cross protective vaccines against streptococcosis in the region.
\end{abstract}

Keyword: Streptococcus agalactiae; Molecular serotyping; Sequence typing; Virulence gene; Pathogenicity 